\title{
Histórico de Violência Intrafamiliar em Pacientes Psiquiátricos
}

Family violence history in psychiatric patients

Histórico de violencia intrafamiliar en pacientes psiquiátricos
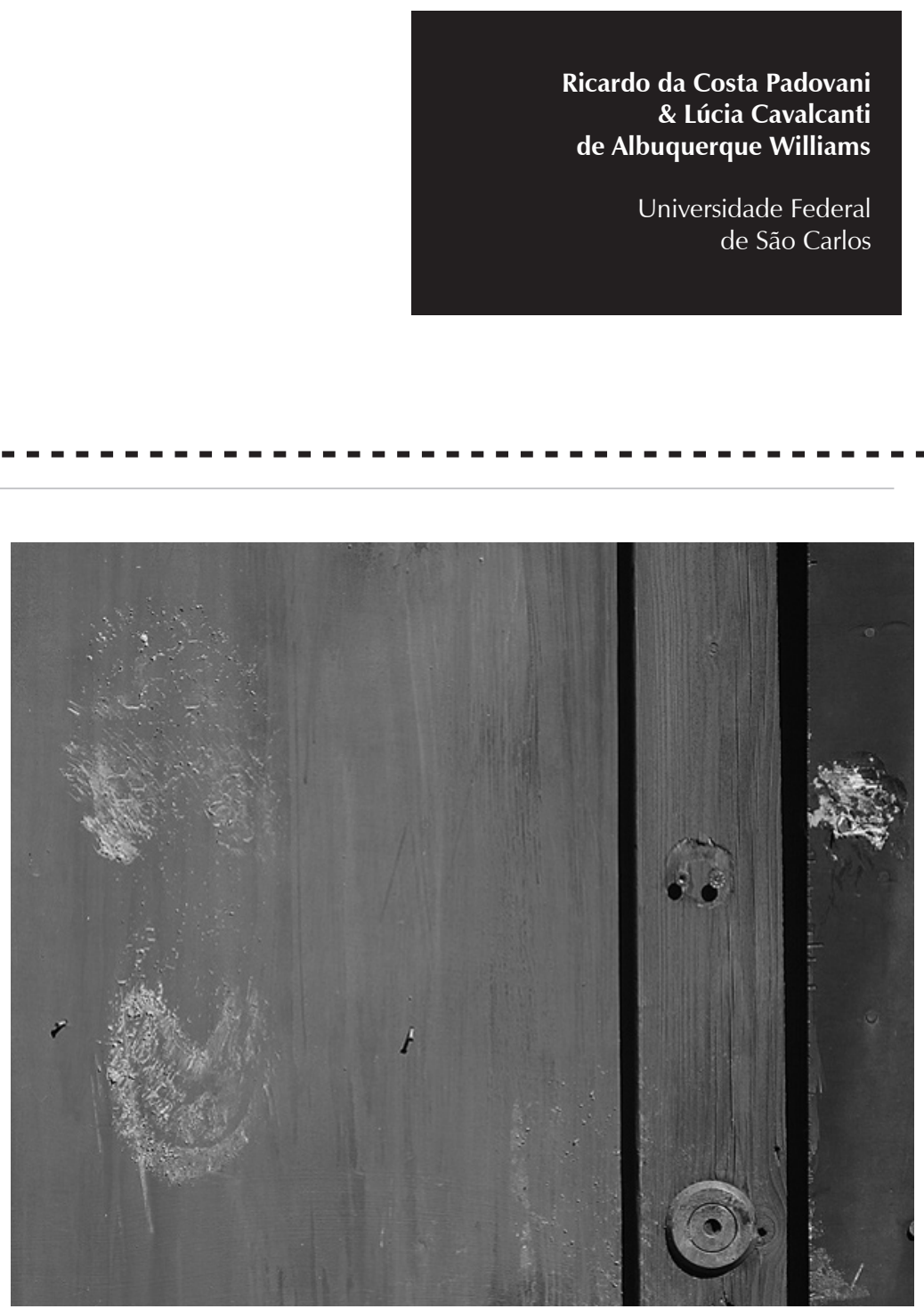
Resumo: O objetivo da presente pesquisa foi caracterizar o histórico de violência intrafamiliar em pacientes psiquiátricos inseridos em tratamento de regime ambulatorial. Pretendeu-se, mais especificamente, identificar o tipo de violência vivenciada pelos usuários. Participaram do estudo 23 pacientes de ambos os sexos, em tratamento ambulatorial. A coleta de dados envolveu a aplicação de um roteiro de entrevista individual, com informações sobre o histórico de internação e violência intrafamiliar e com descrição da infância, dentre outros itens. Apenas um dentre 23 participantes relatou ausência de histórico de violência intrafamiliar, 20 participantes indicaram histórico infantil de maus tratos físicos e psicológicos. Na fase adulta, dos 13 participantes que declararam viver com parceiros/parceiras no presente ou no passado, 8 participantes do sexo feminino relataram agressividade por parte do companheiro e 2 participantes do sexo masculino afirmaram ter agredido as esposas no passado. Com relação à violência sexual, 5 participantes relataram ter experienciado relação sexual forçada por parte do parceiro e um participante relatou ter abusado sexualmente da parceira. Os resultados chamam a atenção pela alta freqüência e pela gravidade de episódios de violência intrafamiliar, que evidenciam a necessidade de se replicar o estudo com amostras maiores e, na prática clínica, investigar tais questões rotineiramente, uma vez que sua esquiva pode contribuir para o processo de adoecimento psíquico.

Palavras-chave: Violência intrafamiliar. Paciente psiquiátrico. Saúde mental. Hospital psiquiátrico.

Abstract: The goal of this study was to characterize the family violence history among psychiatric patients in a mental health outpatient clinic. More specifically, identification of the modality of violence experienced by the patients was intended. Twenty three psychiatric patients who attended the clinic took part of the study. The instrument used to collect data was a semi-structured interview, with questions related to patient's mental health and family violence history, as well as childhood description, among other topics. Only one out of 23 patients reported an absence of family violence history. Twenty participants indicated a history of physical or emotional abuse during childhood. In their adult phase, of the 13 participants who reported having lived with an intimate partner, eight female participants reported aggressive behavior by partners, and two male participants reported perpetrating aggressive episodes to their wives, in the past. In terms of sexual violence, five participants reported having experienced forced intercourse by partners, and one male participant reported having sexually abused his wife. The results call attention to the high frequency and severity of family violence episodes. This phenomenon should be investigated using wider samples, and questions on family violence history should routinely be asked in the clinical practice, as its avoidance during treatment may contribute to poor prognosis.

Keywords: Family violence. Psychiatric patient. Mental health. Psychiatric hospital.

Resumen: El objetivo de la presente pesquisa fue caracterizar el histórico de violencia intrafamiliar en pacientes psiquiátricos inseridos en tratamiento de régimen ambulatorial. Se pretendió, más específicamente, identificar el tipo de violencia vivida por los usuarios. Participaron del estudio 23 pacientes de ambos los sexos, en tratamiento ambulatorial. La recogida de datos implicó en la aplicación de un guión de entrevista individual, con informaciones sobre el histórico de internación y violencia intrafamiliar y con descripción de la infancia, entre otros apartados. Apenas uno entre 23 participantes relató ausencia de histórico de violencia intrafamiliar. 20 participantes indicaron histórico infantil de maltratos físicos y psicológicos. En la fase adulta, de los 13 participantes que declararon vivir con compañeros/compañeras en el presente o en el pasado, 8 participantes del sexo femenino relataron agresividad por parte del compañero, y 2 participantes del sexo masculino afirmaron haber agredido las esposas en el pasado. Con relación a la violencia sexual, 5 participantes relataron haber probado relación sexual forzada por parte del compañero y un participante relató haber abusado sexualmente de la compañera. Los resultados Ilaman la atención por la alta frecuencia y por la gravedad de episodios de violencia intrafamiliar, que evidencian la necesidad de repetir el estudio con muestras mayores y, en la práctica clínica, investigar tales cuestiones rutinariamente, una vez que su esquiva puede aportar para el proceso de enfermedad psíquica.

Palabras-clave: Intrafamiliar. Paciente psiquiátrico. Salud mental. Hospital psiquiátrico. 
Dificuldades

financeiras,

desemprego,

problemas com a

justiça, abuso de

álcool e drogas

apresentam-se

como fatores de

risco, e não devem

ser vistos como

as causas

unilaterais de tal

comportamento

(Sinclair, 1985;

Soares, 1999).

outros estímulos distratores.
A violência intrafamiliar é um fenômeno complexo que desconhece qualquer fronteira de classe social, cultura e nível de desenvolvimento econômico, e pode ocorrer tanto no domínio íntimo do lar como no domínio público e em qualquer etapa da vida (Azevedo \& Guerra, 1995; Saffioti, 1997; Sinclair, 1985; Williams, 2001). Tal violência envolve as modalidades psicológica/ emocional, física e sexual (Saffioti, 1997; Sinclair, 1985). Dificuldades financeiras, desemprego, problemas com a justiça, abuso de álcool e drogas apresentam-se como fatores de risco, e não devem ser vistos como as causas unilaterais de tal comportamento (Sinclair, 1985; Soares, 1999).

A relevância da investigação da violência intrafamiliar entre pacientes psiquiátricos torna-se evidente quando analisamos as seqüelas da violência. Dentre as principais seqüelas, podem-se destacar: processo de vitimização subseqüente, dores crônicas, distúrbio do sono e de alimentação, baixa auto-estima, alto nível de ansiedade e depressão, ideação e tentativas de suicídio, abuso de álcool e drogas e uso não controlado de psicotrópicos (Koss, Bailey, \& Yan, 2003; Saffioti, 1997; Sinclair, 1985; Williams, 2002).

Estudos demonstram que um histórico de episódios de violência intrafamiliar pode favorecer o e/ou agravar os sintomas psiquiátricos, como, por exemplo, os quadros de ansiedade e depressão e as dores crônicas, além de favorecer o abuso de substâncias psicoativas (Coker et al., 2002; Dong et al., 2004; Duran et al., 2004; Edwards et al., 2005; Goldberg \& Garno, 2005; Whitfield, Dube, Felitti, \& Anda, 2005). Olive (2007) verificou que pelo menos $6 \%$ dos pacientes do atendimento de emergência foram vítimas de violência doméstica nos últimos doze meses. Edwards et al. (2005) destacam que a investigação de experiências adversas na infância deveria fazer parte do serviço de rotina médica e, quando constatadas, estas deveriam ser anotadas nos prontuários dos pacientes. Assim, Oliveira (2002) destaca a necessidade de capacitação dos profissionais da área da saúde para a prevenção dos maus tratos infantis.

Nessa mesma direção, o Relatório de Gestão 2003-2006 referente à saúde mental no SUS, no item referente à saúde mental e gênero, relata que a morbidade provocada pela violência doméstica e sexual atinge preferencialmente a população feminina e faz aumentar as internações de mulheres por transtorno de humor, com o crescimento dos transtornos mentais (Ministério da Saúde, 2007).

Como principais sintomas da criança e do adolescente expostos e/ou vitimizados pela violência intrafamiliar, verificam-se: comportamento agressivo (principalmente em garotos), isolamento, passividade, insônia, pesadelos, dores de cabeça, dor de estômago, alergias, comportamento auto-destrutivo, crueldade contra animais, problemas de atenção e memória, fracasso escolar, problemas de aprendizagem, fuga de casa, abuso de drogas e álcool, ideação e tentativa de suicídio, prostituição, gravidez e casamento precoce (Becker-Blease \& Depromce, 2005; Brancalhone \& Williams, 2003; Cherry, 2006; Maldonado \& Williams, 2005; Santos, 2002; Smith, Ireland, \& Thornberry, 2005).

As pesquisas sobre a relação entre um histórico de violência intrafamiliar em pacientes psiquiátricos são recentes. O estudo realizado por Whitfield etal. (2005), por sua vez, apontou evidências da correlação entre um histórico de maus tratos e comportamento alucinatório. 
Os autores alertaram, ainda, para o fato de que essa experiência traumática pode estar subjacente a diversos transtornos psiquiátricos que envolvem o comportamento alucinatório, como, por exemplo, os transtornos de humor, os transtornos dissociativos, a esquizofrenia, o transtorno bipolar e os transtornos decorrentes do uso de substâncias psicoativas.

Nessa mesma direção, Duran e et al. (2004) investigaram a relação entre maus tratos infantis e transtornos psiquiátricos no curso de vida, em um grupo de 234 mulheres americanas de origem indígena, em uma unidade básica de Saúde dos EUA, encontrando alta proporção de participantes (84.2\%) com algum transtorno mental, sendo que $60,7 \%$ das participantes apresentaram critérios diagnósticos para dois ou mais transtornos. Os autores mostraram, ainda, que a prevalência de transtornos mentais aumentava de acordo com os tipos e a severidade de maus tratos. Dentre as 54 participantes que experienciaram todos os tipos de abuso e negligência, $75,9 \%$ apresentaram histórico de abuso e dependência de álcool e drogas, 68,5\%, histórico de transtorno de humor, 83,3\%, transtorno de ansiedade, e $63 \%$, transtorno de estresse pós-traumático.

Ao investigarem a prevalência de comorbidade de transtorno de estresse pós-traumático (TEPT) entre 100 pacientes adultos com transtorno bipolar e a sua relação com categorias de abusos na infância e traumas na vida adulta, Goldberg e Garno (2005) verificaram que 24 dentre 100 participantes (24\%) apresentaram critérios definidores para o TEPT, e um histórico de abuso severo foi identificado em 51 dos casos (51\%). O TEPT ocorreu em aproximadamente um terço dos pacientes com transtorno bipolar que reportaram formas severas de abuso (18/51 pacientes, $35 \%$ ), sendo mais prevalente nesse grupo do que no grupo que não indicou histórico de maus tratos na infância.

Com relação ao perfil do agressor conjugal, a literatura tem apontado características como isolamento social, ciúmes, insegurança, impulsividade, baixa auto-estima, quadros de depressão e ansiedade, podendo ocorrer o uso abusivo de álcool ou drogas, ideações e tentativas de suicídio (Cortez, Padovani, \& Williams, 2005; Gondolf, 1997; Padovani \& Williams, 2002; Rynerson \& Fishel, 1993; Soares, 1999). Estudiosos alertam para o fato de que o agressor conjugal, freqüentemente, apresenta histórico de exposição a maus tratos na infância e/ou adolescência, reproduzindo, o que foi chamado pelos especialistas, de intergeracionalidade da violência (Saffioti, 1997). O impacto da exposição à violência conjugal no comportamento da criança e do adolescente foi discutido por Ormeño e Williams (2006) e Smith et al. (2005), entre outros.

Confirmando os efeitos nocivos da violência, Adeodato, Carvalho, Siqueira e Souza (2005) mostraram que, de 100 mulheres que sofreram violência por parte de seus parceiros e prestaram queixa na Delegacia de Defesa da Mulher do Ceará, 72\% apresentavam quadros depressivos, $78 \%$ tinham sintomas de ansiedade e insônia, $39 \%$ pensaram em suicídio e $24 \%$ passaram a fazer uso de ansiolítico após o início da agressão. Adicionalmente, foi constatado pelos autores que o uso de ansiolíticos aumentou independentemente do tipo, freqüência e tempo de agressão.

A necessidade de se realizar intervenções sistemáticas com mulheres vitimizadas nos diferentes setores da área da saúde foi apontada por Schraiber, D'Oliveira, França e Pinho (2002), indicando que a questão da violência contra a mulher raramente aparece nos diagnósticos e nas condutas 
realizados nos serviços de saúde. Os mesmos autores destacaram, ainda, que a violência intrafamiliar é uma realidade comum entre as usuárias do serviço de saúde estudado. Segundo o estudo, em cada três usuárias, uma apresenta histórico de violência intrafamiliar, o que é preocupante. Ainda nesse contexto, é preocupante a afirmação de Santos (2003) de que o profissional da saúde não se sente apto para tratar da questão da violência intrafamiliar e que os serviços não estão preparados para atender, diagnosticar e conduzir casos dessa natureza.

A ausência de diagnóstico de presença de violência direcionada à mulher nos serviços de saúde mental foi discutida por Koss et al. (2003), que apontaram que, apesar da alta incidência de mulheres vitimizadas nos serviços de saúde mental, a maioria delas não relata a questão da violência intrafamiliar aos profissionais da saúde, utilizando recursos próprios para lidar com as adversidades provenientes de uma relação abusiva. Tal estudo mostrou, também, que o histórico de violência intrafamiliar é raramente analisado no processo de avaliação do paciente. Como possíveis hipóteses para tal omissão, os autores destacam: as características pessoais do profissional, especialmente, a falta de repertório, o medo de ofender o paciente e as crenças errôneas sobre violência intrafamiliar. Essa realidade deixa evidente a outra face da precariedade dos serviços de saúde brasileiros.

Em contrapartida, conforme mostrou Minayo (2002), esforços encorajadores foram observados entre médicos pediatras no que se refere ao enfrentamento dos maus tratos infantis. Em tal artigo, a autora relata a decisão da Sociedade Brasileira de Pediatria de adotar a questão da violência contra a criança e a adolescência como mais uma de suas responsabilidades.
Paralelo à sensibilização de médicos e equipe de saúde sobre a problemática da violência intrafamiliar, reforça-se a necessidade de se investir na produção de materiais que venham aprimorar o conhecimento dos profissionais envolvidos, o que auxiliaria no processo de diagnóstico e no desenvolvimento de programas de prevenção. Minayo (2002) reconhece, também, que o serviço público de saúde brasileiro precisa melhorar consideravelmente para atingir os serviços de prevenção e promoção desenvolvidos, por exemplo, no Canadá e nos Estados Unidos. A relevância e a necessidade de se desenvolver tais programas ficam evidentes quando o foco do serviço de saúde são indivíduos com transtornos mentais.

Posturas semelhantes foram verificadas no estudo desenvolvido por Deslandes, Gomes e Silva (2000), que investigaram os casos de violência intrafamiliar contra a mulher em relação ao atendimento emergencial por causas externas em dois hospitais públicos situados no Município do Rio de Janeiro. Paralelamente, os autores buscaram caracterizar as vítimas e o atendimento prestado, além de analisar as circunstâncias em que ocorreram esses eventos. Os dados mostraram que, na maioria dos casos, o agressor era o marido (56,9\%), acompanhado dos ex-maridos $(6,9 \%)$, namorados $(4,2 \%)$ e ex-namorados $(1,4 \%)$, sendo as agressões por espancamento as mais comuns $(70,4 \%)$, direcionadas sobretudo para a região da cabeça e da face. O estudo mostrou, ainda, que a maioria das agressões ocorria no interior da residência (83,3\% dos casos). Outro dado interessante apontado pelo estudo foi que em apenas 5 dos 72 casos estudados a vítima revelou temor ou constrangimento em relatar o acontecimento ao profissional de saúde.

Morrison, Allan e Grunfeld (2002) compararam a taxa de violência intrafamiliar identificada 
com base no questionamento direto e a registrada pelas usuárias de um serviço de emergência desenvolvido no Royal Victoria Hospital, em Montreal (Canadá), instituição afiliada à Universidade McGill. Na etapa referente à análise de prontuários, foram analisados 1000 prontuários e identificados quatro casos. Na segunda etapa, que envolveu o desenvolvimento e a aplicação de um questionário aplicado em 302 pacientes, foram identificados 43 casos $(14,2 \%)$. O presente dado ilustra a eficiência do questionamento direto no processo de identificação da violência intrafamiliar. Presume-se ainda que, em muitas situações, essa fase constitua o primeiro momento de ajuda para essas mulheres vitimizadas. Os autores afirmam que a crença de que o questionamento sobre a violência intrafamiliar possa ofender ou causar constrangimento às pacientes não apresenta consistência com os dados da realidade, argumento também defendido por Koss et al. (2003). Ainda nessa direção, apoiado em pesquisas desenvolvidas na Nova Zelândia, Read (2007) demonstra a relevância do questionamento do histórico de violência em serviços de saúde mental e como o processo de questionamento aumenta o número de casos identificados. O autor destaca, também, a necessidade de capacitação do profissional nesse processo de investigação do histórico de violência.

Diante do impacto da violência intrafamiliar na saúde mental dos indivíduos elucidado pela literatura (Coker et al., 2002; Cherry, 2006; Duran et al., 2004; Maldonado \& Williams, 2005; Olive, 2007; Saffioti, 1997; Sinclair, 1985; Smith et al., 2005; Whitfield et al., 2005; Williams, 2002), foram levantadas, no presente estudo, as seguintes questões: pacientes com histórico de internação psiquiátrica apresentariam um histórico de maus tratos infantis e/ou de violência intrafamiliar na vida adulta?
Em caso afirmativo, qual o tipo de violência experienciada?

Assim, o presente estudo teve como objetivo caracterizar o histórico de violência intrafamiliar em pacientes com internação psiquiátrica em regime ambulatorial. Mais especificamente, pretendeu-se identificar o tipo de violência vivenciada (emocional, física e sexual), seja durante a infância, seja na idade adulta, em pacientes psiquiátricos de ambos os sexos, usuários de um serviço de saúde mental.

\section{Método}

Triagem dos participantes

Os critérios utilizados para selecionar possíveis participantes foram: estar em alta hospitalar, participar do programa das oficinas terapêuticas da instituição e apresentar avaliação psiquiátrica favorável. No presente estudo, avaliação psiquiátrica favorável foi definida como orientação no tempo e no espaço, discurso organizado de ordem lógica e ausência de alucinações. O próprio processo de alta constitui uma variável favorável ao ajustamento psicossocial do paciente.

\section{Participantes}

Participaram do estudo 23 pacientes psiquiátricos em tratamento ambulatorial de um hospital psiquiátrico de uma cidade do interior do Estado de São Paulo, sendo 13 participantes do sexo feminino e 10 do sexo masculino.

Local

A coleta de dados foi realizada na sala destinada ao atendimento psicológico da instituição psiquiátrica mantida pelo 
Sistema Único de Saúde (SUS). As oficinas terapêuticas tinham capacidade para o atendimento de 30 usuários. A equipe das oficinas era composta por um psicólogo, uma assistente social, uma psiquiatra, um clínico geral e dois monitores.

\section{Consentimento Livre e Esclarecido}

Os pacientes selecionados foram informados sobre os propósitos do estudo, o sigilo da identidade e da imagem, os cuidados éticos assegurados, a não obrigatoriedade em participar do estudo e a possibilidade de divulgação dos resultados obtidos em eventos científicos. O projeto foi submetido ao Comitê de Ética em Pesquisa em Seres Humanos da UFSCar, tendo sido aprovado (Parecer no. 054/2005).

Instrumento de coleta de dados

Foi utilizado um roteiro de entrevista individual semi-estruturado baseado no modelo de entrevista com crianças expostas à violência conjugal (Williams, 2004) e no instrumento descrito no estudo de Martin, Bergen, Richardson e Allison (2004). O roteiro abordava os seguintes itens: data de nascimento, escolaridade, constituição familiar, histórico de internação, uso de substâncias psicoativas, histórico de violência intrafamiliar (psicológica, física e sexual), descrição da infância e auto-avaliação.

\section{Procedimento}

Inicialmente, o primeiro autor, que também atuava como psicólogo da mesma instituição, agendou uma reunião com o diretor e sua equipe de trabalho para a apresentação e a apreciação do projeto de pesquisa em questão, tendo recebido parecer favorável. Paralelamente, o projeto foi encaminhado ao Comitê de Ética em Pesquisa em Seres Humanos da UFSCar.
Antes de aplicar a entrevista, o primeiro autor consultava o prontuário para avaliar a evolução do paciente nas últimas duas semanas. Assinado o Termo de Consentimento Livre e Esclarecido, o primeiro autor aplicava, individualmente, o roteiro de entrevista semi-estruturado anotando as respostas dos participantes. A entrevista era conduzida em uma única sessão, com média de 50 minutos de duração. No momento subseqüente ao término da entrevista, o pesquisador conduzia uma sessão de psicoterapia com o objetivo de garantir o bem-estar psicossocial do participante. É importante ressaltar que todos os participantes estavam recebendo sistematicamente atendimento psiquiátrico, psicológico e acompanhamento da Assistência Social.

\section{Análise de dados}

Os resultados foram analisados quantitativamente e qualitativamente. A análise quantitativa envolveu a mensuração da freqüência do tipo de diagnóstico, o número de internações, a freqüência de maus tratos e a exposição à violência familiar tanto na infância quanto na vida adulta de cada participante. Para analisar a freqüência de maus tratos infantis e a vitimização na vida adulta, foram criadas categorias de acordo com o tipo de violência relatada pelos participantes durante a entrevista. A análise qualitativa fundamentou-se na análise do auto-relato dos participantes durante a coleta de dados, particularmente no que se refere ao histórico de violência conjugal, maus tratos na infância, descrição da infância e auto-avaliação.

\section{Resultados}

Os resultados serão apresentados em cinco categorias: 1. Caracterização sociodemográfica dos participantes; 2. Informações sobre o 
transtorno mental; 3. Histórico de violência intrafamiliar; 4. Descrição da infância; 5. Autoavaliação. Para facilitar a identificação do gênero de cada participante, será utilizada a sigla $\mathrm{P}$ para identificar os participantes do sexo masculino e PP para participantes do sexo feminino.

\section{Informações sociodemográficas}

Dentre os 23 participantes, apenas uma participante (PP14) não apresentou histórico de internação psiquiátrica. PP14 procurou o serviço por não conseguir desempenhar suas atividades profissionais, pessoais e sociais em função de estar apresentando sintomas mistos de ansiedade e depressão. A Tabela 1, a seguir, traz a descrição dos dados demográficos dos participantes do estudo.

Tabela 1. Caracterização dos participantes.

\begin{tabular}{lllllll}
\hline Participante & Idade & Sexo & Escolaridade & Estado civil & Filhos & Reside \\
\hline P1 & 37 & M & EFI & Casado & 5 & Esposa \\
PP2 & 40 & F & EMI & Solteira & - & Pais \\
P3 & 49 & M & EFI & Separado & 1 & Sozinho \\
PP4 & 41 & F & EFI & União estável & 4 & Parceiro \\
P5 & 44 & M & EFI & Solteiro & 2 & Sozinho \\
PP6 & 73 & F & EFI & Casada & 4 & Marido \\
P7 & 55 & M & EMC & Viúvo & 2 & Sozinho \\
PP8 & 59 & F & EFI & Viúva & 3 & Filho \\
PP9 & 53 & F & EFI & União estável & 3 & Parceiro \\
PP10 & 33 & F & EFI & Solteira & 1 & Pais \\
PP11 & 50 & F & EFI & Casada & 1 & Marido \\
P12 & 43 & M & Analfabeto & Casado & 1 & Esposa \\
PP13 & 59 & F & EFI & Viúva & 10 & Sozinha \\
PP14 & 28 & F & EMC & Casada & 1 & Mãe \\
P15 & 51 & M & EFI & Solteiro & - & Amigos \\
P16 & 26 & M & EFI & Solteiro & - & Pais \\
P17 & 50 & M & Analfabeto & Solteiro & - & Irmãos \\
PP18 & 38 & F & EFI & Casada & 2 & Marido \\
P19 & 41 & M & EFI & Solteiro & - & Irmã \\
P20 & 45 & M & EFI & Solteiro & - & Amigo \\
PP21 & 39 & F & EFI & Solteira & - & Pais \\
PP22 & 45 & F & EMC & Solteira & 3 & Filhos \\
PP23 & 28 & F & ESI & Solteira & - & Sozinha \\
\hline
\end{tabular}

* EFI (ensino fundamental incompleto), EMI (ensino médio incompleto), EMC (ensino médio completo), ESI (ensino superior incompleto).

Os dados da Tabela 1 mostram que a idade dos participantes variou de 26 a 73 anos. 3 participantes tinham idade entre 26 e 36 anos; 10 tinham entre 37 e 47 anos; 5, entre 48 e 58 anos, 2, entre 59 e 69, e um, 73 anos. Com relação ao estado civil, 6 participantes (4 do sexo feminino e 2 do sexo masculino) eram casados, 2 viviam em regime de união estável (ambos do sexo feminino), 1 estava separado, 3 eram viúvos ( 2 do sexo feminino e 1 do sexo masculino) e 11 declararam-se solteiros.

Com relação à escolaridade, verifica-se, na Tabela 1, que apenas dois participantes relataram nunca haver freqüentado a escola (P12, P17). Todos os participantes que freqüentaram a escola haviam interrompido os estudos. A participante 23 foi a que apresentou maior escolaridade (3으 grau incompleto). PP23 relatou que estava cursando Letras em uma Universidade Estadual, tendo interrompido o estudo no quarto semestre, após inúmeras faltas e repetências. Nenhum participante exercia atividade profissional remunerada. A maioria dos participantes $(65 \%)$ relatou ter filhos, sendo que a participante 13 foi a que apresentou o maior número: 10 filhos. 
Quanto à moradia, sete participantes (P1, PP4, PP6, PP9, PP11, P12, PP18) afirmaram que moravam com parceiros/parceiras, cinco (P3, P5, P7, PP13, PP23) relataram que moravam sozinhos, cinco moravam com os pais (PP2, PP10, PP14, P16, PP21), dois com irmãos (P17, P19), outros dois com os filhos (PP8, PP22) e mais dois (P15, P20) moravam em uma das pensões protegidas (unidades habitacionais supervisionadas mantidas pela instituição).

\section{Informações diagnósticas}

O manual de diagnóstico de transtorno mental utilizado pela instituição era o CID-10 (1993).

A Tabela 2 ilustra as categorias diagnósticas encontradas no estudo.

Tabela 2. Categorias de diagnóstico dos participantes.

\begin{tabular}{llc}
\hline Diagnóstico & Participantes & Total \\
\hline Alucinose orgânica & P17 & 1 \\
Esquizofrenia & P1, PP4, P19, P20, PP23 & 5 \\
Transtorno depressivo & PP13, PP18 & 2 \\
Transtorno misto de ansiedade & PP2, PP14 & 2 \\
e depressão & & \\
Transtorno afetivo bipolar & PP8, PP9, PP11, P12, P16, PP21, PP22 & 7 \\
Síndrome de dependência & P3, P5, PP6, P7, PP10, P12, P15 & 7 \\
\hline
\end{tabular}

É possível verificar, na Tabela 2, a predominância do transtorno definido como síndrome de dependência e transtorno afetivo bipolar (sete ocorrências), seguido da esquizofrenia (cinco ocorrências). Em menor freqüência, verifica-se o transtorno definido como alucinose orgânica (P17). Adicionalmente, chamam a atenção os transtornos depressivos e mistos de ansiedade e depressão, observados exclusivamente entre participantes do sexo feminino, a predominância da síndrome de dependência entre participantes do sexo masculino, e do transtorno afetivo bipolar entre participantes do sexo feminino.

A Tabela 3 ilustra a freqüência de internação de cada participante.

Tabela 3. Freqüência de internação de cada participante.

\begin{tabular}{llc}
\hline Internações & Participantes & Total \\
\hline $1-5$ & PP2, PP6, P7, PP9, PP10, PP11, PP21, & 12 \\
& PP13, P16, P17, PP18, P19 & \\
$6-12$ & P3, PP8, P12 & 3 \\
$15-21$ & P1, PP4, P5, P15, P20, PP22, PP23 & 7 \\
\hline
\end{tabular}

Nota: PP14 não apresentava histórico de internação psiquiátrica, apenas participava das oficinas terapêuticas.

Segundo os dados da Tabela 3, participantes com internações que variavam em freqüência de 1 a 5 compuseram o maior grupo (12 participantes), seguidos dos participantes com 15 a 21 internações (7 participantes). Ogrupo com menor ocorrência, nesse sentido, foi o de 6 a 12 internações (3 participantes). Observa-se a maioria de participantes do sexo feminino no grupo de 1 a 5 internações (8 participantes). Nas demais categorias, verificou-se maior freqüência de participantes do sexo masculino. 
Cabe ressaltar que P5 foi o paciente que apresentou o maior número de internações (21 internações), sendo seu diagnóstico a síndrome de dependência. Em seguida, encontram-se os participantes 4 e 22 (com 20 internações), acompanhados pelos participantes 15 e 23 (com 19 internações). Quatro participantes indicaram apenas uma internação (P7, PP21, PP13, P16).

\section{Histórico de violência intrafamiliar Infância}

A Tabela 4 ilustra as principais categorias de agressão física e psicológica sofridas pelos participantes na infância.

Tabela 4. Categorias de maus tratos infantis sofridos na infância.

\begin{tabular}{lll}
\hline Categorias & Participantes & Total \\
\hline Agredir com chinelo & PP2, PP21, PP23 & 2 \\
Puxar orelha, cabelo, arranhar o braço & P1, PP2, P17, P3, PP8, PP13, PP22 & 7 \\
Bater na cabeça com escova de cabelo & PP18 & 1 \\
Agredir com cinto, vara & PP2, PP4, PP10, P20, PP18, P5 & 6 \\
Dar banho de salmoura depois de bater & PP4, PP8, PP18 & 3 \\
Estapear, socar, chutar, & P1, P3, P7, PP10, PP12, & 8 \\
atirar objetos & PP13, P20, PP22 & \\
Ficar ajoelhado no milho & P1 & 1 \\
Tirar a roupa e agredir nu & P20 & 1 \\
Ameaçar com faca & P12, P20 & 2 \\
Cortar cabelo com facão & PP8 & 1 \\
Exibir revólver dentro de casa & P3 & 1 \\
Agredir com objetos: chicote, & P1, P3, P5, PP6, PP8, P15, & \\
fio de ferro, corda, cano, pau & P16, P17, PP21 & 9 \\
Colocar forcado no pescoço & P1 & 1 \\
\hline
\end{tabular}

Dos 23 participantes, apenas três participantes (PP14, PP11, P19) não indicaram histórico de maus tratos na infância. É interessante destacar que tais participantes tiveram o menor número de internação. Nenhum participante indicou histórico de violência sexual nessa fase.

Os dados mostram que os participantes foram vítimas de mais de uma modalidade de violência na infância, sendo que P3 foi o participante que sofreu a maior variabilidade de agressões (seis modalidades), acompanhado de P1 e PP8 (cinco modalidades). Tais modalidades permitem constatar que a agressão com objetos (chicote, fio de ferro, cano, pau e corda) foi a modalidade de violência mais freqüente, seguida de tapas, socos, chutes e atirar objetos. Já em menor freqüência, apresentam-se as seguintes modalidades: tirar a roupa da criança e bater, cortar cabelo com facão e colocar forcado no pescoço.

Agredir com chinelo, arranhar o braço, bater na cabeça com cabo de escova de cabelo, agredir com vara, dar banho de salmoura depois de bater, atirar objetos, cortar cabelo com facão, agredir com mangueira ou cano foram modalidades de agressões físicas apontadas exclusivamente por participantes do sexo feminino. Já as modalidades apontadas exclusivamente por participantes do sexo masculino foram: ficar ajoelhado no milho atrás da porta, tirar a roupa e receber agressões nu, desferir paulada nas costas e na cabeça, agredir com chicote, com corda, galho de árvore e colocar forcado no pescoço. 


\section{Descrição da infância}

Quando questionados sobre a infância, 47,8\% dos participantes destacaram-na como tendo sido "boa" ou "feliz", sendo esse período caracterizado por brincadeiras, passeios, freqüentar a escola, entre outras atividades. Entretanto, apesar de mencionar que sua infância tinha sido feliz, P23 afirmou que o pai a agredia e a humilhava (dizia que era "vagabunda") e que ler "não dava nada". Em contrapartida, 52,1\% (P1, P3 PP6, PP8, PP9, PP10, PP12, PP13, P16 PP18, P20, PP22) descreveu a infância como tendo sido "difícil", "triste", "tumultuada", "péssima" e de "muito trabalho".

Os participantes deram como justificativa para uma infância difícil os momentos das agressões físicas, a presença de violência dentro de casa, não ter local onde residir, dormir embaixo de pontes e "no mato", nunca ter ganhado um presente e o distanciamento afetivo dos pais. Seguem abaixo exemplos de relatos sobre a infância: "Difícil. Eu apanhei bastante, não saía de casa. Só trabalhava" (P1); "Foi pancadaria e brigas" (PP9). PP22 mencionou que seu pai "sumiu" quando ela tinha quatro anos e que sua mãe era agressiva: desferia socos no rosto, jogava objetos em sua direção e arranhava seu braço. Todos os relatos deixam evidentes os efeitos nocivos dos maus tratos sofridos na infância (violência física e negligência) nas lembranças dos participantes.

\section{Fase adulta: vida conjugal}

13 participantes declararam viver ou ter vivido com parceiros/parceiras (56,5\%), 8 participantes (34.6\%) relataram que seus parceiros eram agressivos (PP4, PP6, PP8, PP9, PP13, PP11, PP14, PP22); 2 participantes (8.6\%) afirmaram ter agredido as esposas no passado (P1, P12) e apenas 3 participantes (PP2, P7, PP18) relataram nunca terem agredido ou terem sido vítimas de agressões físicas ou verbais do cônjuge.

Com relação à violência sexual, cinco participantes do sexo feminino (PP4, PP8, PP9, PP11, PP13) relataram que experienciaram relação sexual forçada por parte do parceiro. Um participante do sexo masculino (P1) afirmou que manteve relações sexuais com a parceira sem o consentimento dela.

A Tabela 5 ilustra as categorias de violência física e psicológica provocadas pelo parceiro relatadas pelas participantes do sexo feminino.

Tabela 5. Categorias de violância provocadas pelo parceiro apresentadas relatadas pelas participantes durante a vida conjugal.

\begin{tabular}{llc}
\hline & Participantes & Total \\
\hline $\begin{array}{l}\text { Empurrar, puxar cabelo, } \\
\text { chutar, socar, estrangular }\end{array}$ & PP4, PP6, PP9, PP13,PP8, PP14 & 6 \\
Ameaçar com arma & PP4, PP8, PP13, PP22 & 4 \\
Fazer sexo forçado & PP4, PP9, PP11, PP13, PP8 & 5 \\
Jogar contra a parede & PP6 & 1 \\
Morder & PP13 & 1 \\
Bater com pedaço de pau & PP9 & 1 \\
Jogar água quente & PP9 & 1 \\
Colocar faca no pescoço & PP8 & 1 \\
\hline
\end{tabular}


As agressões empurrar, puxar cabelo, chutes e socos foram as categorias mais destacadas pelas participantes (6). Manter relações sexuais com a parceira sem o consentimento desta também se apresentou com alta freqüência, tendo sido apontado por cinco participantes. Observa-se, ainda, na Tabela 5, a presença de mais de uma modalidade de violência empregada pelos parceiros. A gravidade da violência física pode ser verificada pelas categorias "jogar água quente", "bater com pedaço de pau" e "ameaçar com arma".

Ainda em relação às agressões ocorridas na vida conjugal, o participante 1 relatou que puxava o cabelo, dava murros e chutes na esposa, e que tinha forçado a mesma a manter relações sexuais com ele. P12 relatou que, em certa ocasião, empurrara a esposa. Afirmou ainda que a esposa tinha medo de suas reações quando ficava nervoso. Segundo o próprio participante, quando sua esposa começava a deixá-lo nervoso, advertia: "É melhor você ficar quieta", para não agredila. P12 costumava chutar os objetos da casa, relatando que, em certa ocasião, havia quebrado o tanque e a mesa de casa quando nervoso.

É interessante observar que apenas um participante (P19) não apresentou histórico de violência intrafamiliar. Tal participante tinha 41 anos, era solteiro, vivia com a irmã e tinha diagnóstico de esquizofrenia, com três internações.

\section{Auto-avaliação}

Ao serem questionados sobre sua atual situação, suas expectativas futuras, as possíveis explicações para seu estado de saúde físico e mental e os sentimentos (positivos e negativos) no momento presente, 10 participantes (43\%) apresentaram respostas positivas e indicativas de melhoria do bem-estar psicossocial. P20 associou seu bem-estar à ausência de brigas. Os demais participantes (57\%) relataram sensações negativas e traumáticas. P7, por exemplo, afirmou: "Sinto falta de alguma coisa. Eu vivo brincando, mas, por dentro, eu não sou feliz". PP18 argumentou que se sentia "péssima, sem vida, sem prazer para a vida" e que "se sentia um vegetal".

\section{Discussão}

Todos os participantes aceitaram participar da pesquisa e em nenhum momento optaram por interromper a entrevista, deduzindo-se, então, que não a consideraram aversiva. $\mathrm{O}$ fato de o pesquisador ser também o psicólogo da instituição possivelmente contribuiu para a coleta de dados da pesquisa. Corroborando os dados da literatura (Deslandes et al., 2000; Koss et al., 2003; Morrison et al., 2002; Sinclair, 1985), pode-se afirmar que a investigação do fenômeno da violência não causou constrangimentos aos participantes. O relato da participante 13 e do participante P15, respectivamente, veio confirmar essa hipótese: "eu achei muito bom" (referindo-se à aplicação do roteiro de entrevista), "gostei de ter participado" (PP13); "achei muito bom. É bom conversar. Se a gente fica reprimindo as coisas, a gente explode" (P15).

O perfil dos participantes foi caracterizado por baixa escolaridade e condições socioeconômicas desfavorecidas, características comuns da população psiquiátrica atendida pelo Sistema Único de Saúde (SUS). Confirmados os achados da literatura, pode-se afirmar que a presença violência intrafamiliar, no passado ou no presente, apresentou-se como um fator de risco para a saúde mental (Nolen-Hoeksema \& Keita, 2003; Ormeño \& Williams, 2006; Saffioti, 1997; Sinclair, 1985; Whitfield et al., 2005; Williams, 2002). 
A ausência de histórico de violência intrafamiliar ao longo do curso de vida em apenas 1 dentre 23 participantes (P19) reforça a necessidade de se investigar rotineiramente questões associadas à violência intrafamiliar em pacientes psiquiátricos. Como apontaram Koss et al. (2003), apesar da alta incidência de mulheres vitimizadas nos serviços de saúde mental, a maioria delas não relata ao profissional da saúde sua situação de vítima no próprio lar, utilizando para isso recursos próprios para lidar com as adversidades, recursos que, na maioria das vezes, se mostram insuficientes para interromper $\mathrm{o}$ ciclo de vitimização ou agressão.

Um agravante observado na população estudada foi a severidade da agressão identificada (cortar cabelo com o facão, colocar forcado no pescoço, jogar água quente) e a presença de mais de uma modalidade de agressão física (socar, chutar, ficar de joelho no milho) entre os participantes.

Outro estressor de extrema gravidade relatado pelo presente estudo foi que, adicionalmente à violência física e psicológica, cinco participantes do sexo feminino revelaram a presença da violência sexual na vida conjugal, sendo que um participante do sexo masculino afirmou ter mantido relações sexuais com a parceira sem o consentimento desta. As implicações da presença de estressores associados à violência sexual para a saúde física e mental vêm sendo analisadas por estudiosos da área (ver, por exemplo, Rodrigues, Brino, \& Williams, 2006).

A síndrome de dependência, predominante na população estudada e caracterizada pelo abuso de substâncias alcoólicas e/ou drogas, vem reforçar os dados da literatura referente ao abuso de tais substâncias entre agressores e vítimas (Adeodato et al., 2005; Deslandes et al., 2000; Duran et al., 2004; Sinclair,
1985; Whitfield et al., 2005). Além de se tornar um agravante para o comportamento do agressor, o uso de substâncias psicoativas pode, também, atuar como recurso para a supressão da dor física e do sofrimento psíquico da vítima.

Retomando os resultados encontrados por Adeodato et al. (2005), o uso de ansiolíticos aumentou entre as mulheres que haviam sido agredidas pelos parceiros. Pode-se especular que a presença exclusiva de transtornos depressivos e de ansiedade e depressão entre participantes do sexo feminino possa estar, em parte, associada à relação assimétrica de poder entre gêneros como também à sensação de impunidade ao comportamento do agressor do homem, aspectos discutidos por Padovani e Williams (2002), Saffioti (1997), Sinclair (1985) e Williams (2001).

Dentre as possíveis variáveis ambientais correlacionadas com a alta taxa de internação observada na amostra estudada, a literatura destaca: a não adesão ao tratamento ambulatorial e medicamentoso, a presença de episódios de violência no lar, a topografia do comportamento violento, as conseqüências nocivas decorrentes do histórico de violência, a falta de envolvimento dos familiares no tratamento do paciente, a falta de repertório dos familiares no processo de identificação precoce de indicadores comportamentais de desajustamento e a presença de problemas psiquiátricos em familiares, além das próprias características decorrentes do transtorno (Duran et al., 2004; Koss et al., 2003; Melo \& Guimarães, 2005; Whitfield et al., 2005).

O presente estudo evidencia o fato de que a investigação do histórico de agressões no âmbito familiar de pacientes psiquiátricos deveria ser um procedimento de rotina no serviço público de hospitais psiquiátricos, para que esse aspecto não seja subdiagnosticado. 
É possível que a existência de graves conflitos intrafamiliares se apresente como o fator desencadeador de uma série de contingências aversivas e favorecedoras do adoecimento psíquico. Adicionalmente, a não informação do impacto dessa realidade e a não instrumentalização dos pacientes sobre as estratégias a serem empregadas no processo de resolução desse problema pode explicar a alta reincidência de internação em hospitais psiquiátricos.

\section{Conclusões}

Este trabalho teve como objetivo caracterizar o histórico de violência intrafamiliar entre pacientes psiquiátricos em tratamento de regime ambulatorial e pretendeu, mais especificamente, identificar o tipo de violência vivenciada. Os resultados indicaram um alto índice de violência intrafamiliar entre os participantes: apenas 1 entre 23 participantes não relatou tal tipo de violência. Torna-se imperativo, portanto, investigar tal fenômeno em pacientes inseridos em unidades psiquiátricas, uma vez que não trabalhar com essas questões no processo de tratamento pode estar contribuindo para o adoecimento psíquico.

Especula-se aqui que, se no processo de tratamento fossem trabalhadas questões referentes à violência contra a mulher e contra a criança, tal medida favoreceria uma redução dos níveis de reincidência de internação e de abandono do tratamento.

Para se obter mais dados sobre tal processo, é necessária a capacitação de profissionais da área da saúde sobre a problemática da violência intrafamiliar, aspecto já apontado pela literatura (Minayo, 2002; Santos, 2003). A ausência de avaliação do histórico de violência intrafamiliar no protocolo de atendimento de instituições de saúde mental contrasta com os dados aqui obtidos, o que reforça a necessidade de debate e de reflexão pelos profissionais da área de saúde e de órgãos gestores, como defendido por Minayo (2002) e Santos (2003).

No presente estudo, chamou a atenção a baixa escolaridade dos usuários de serviços psiquiátricos no Sistema Único de Saúde. Trata-se de uma variável de extrema relevância para o planejamento do tratamento desses usuários. A falta de informação e de repertório nessa clientela pode favorecer a manutenção de quadros de violência na dinâmica intrafamiliar, portanto, ações psico-educativas no processo de tratamento poderiam constituir estratégias eficazes no processo de conscientização e, em conseqüência, de mudança comportamental. Criar condições de acessar as crenças relacionadas à violência, suas concepções de violência intrafamiliar, as fontes de recursos, tanto no próprio indivíduo como no seu meio social, e a orientação quanto aos seus direitos como cidadãos são aspectos que merecem ser discutidos e analisados pela equipe profissional.

Pode-se inferir que a presença de mais de uma modalidade de violência e gravidade observada entre os participantes do estudo potencialize os efeitos danosos da violência intrafamiliar na saúde mental dos vitimizados, como, por exemplo, o aumento do quadro de ansiedade e depressão e o abuso de substâncias psicoativas, preocupações apontadas por estudiosos da área (Coker et al., 2002; Duran et al., 2004; Edwards et al., 2005; Whitfield et al., 2005; Olive, 2007).

Ainda nessa mesma perspectiva, o uso de objetos, como, por exemplo, facão, corda e cano, vêm ilustrar o potencial ofensivo da agressão sofrida pelos participantes na infância. Além de o profissional ter 
que saber abordar questões referentes à violência intrafamiliar, ele deve, também, ser capaz de agir conforme as disposições do Estatuto da Criança e do Adolescente (Brasil, 1990), quando suspeitar da ocorrência de maus tratos infantis no âmbito familiar do paciente.

Pode-se elaborar a hipótese que, diante de um estressor grave como a violência intrafamiliar, o indivíduo com vulnerabilidade biológica possui maior probabilidade de apresentar sintomas psiquiátricos. A pergunta decorrente e de investigação complexa envolve saber se o transtorno mental teria se manifestado caso não tivesse existido o estressor violento. Ou ainda, se a violência não existisse, o prognóstico e o processo de reabilitação psicossocial seriam os mesmos? Outros estudos poderão continuar a investigar essa relação utilizando amostras maiores.

\section{Ricardo da Costa Padovani *}

Psicólogo, Doutor em Educação Especial pela UFSCar e pesquisador do Laboratório de Análise e Prevenção da Violência (LAPREV) da Universidade Federal de São Carlos/UFSCar

\section{Lúcia Cavalcanti de Albuquerque Williams}

Psicóloga, Doutora em Psicologia Experimental, Professora Titular do Departamento de Psicologia da Universidade Federal de São Carlos, coordenadora do LAPREV

\section{* Endereço para envio de correspondência:}

Ricardo da Costa Padovani

Fone: (16) 3335-2696 / (16) 9777-1975

Rua: Voluntários da Pátria, 2717, ap. 161

CEP: 14.801-320 - Araraquara - SP.

E-mail: ricardopadovani@yahoo.com.br

Recebido 23/07/2007 Reformulado 06/05/2008 Aprovado 07/06/2008

Adeodato, V. G., Carvalho, R. R., Siqueira, V. R., \& Souza, F. C. M. (2005). Qualidade de vida e depressão em mulheres vítimas de seus parceiros. Revista de Saúde Pública, 39(1), 108-113.

Azevedo, M. A., \& Guerra, V. N. A. (1995). Violência doméstica na infância e adolescência. São Paulo: Robe.

Becker-Blease, K. A., \& Depromce, A. P. (2005). Child victimization, cognitive functioning and academic performance. In K. A. Kendall-Tackett \& S. M. Giacomoni (Eds.), Child victimization: Maltreatmentm bullying and dating violence, prevention and intervention (pp. 1-13). New Jersey: Civic Research Institute.

Brancalhone, P. G., \& Williams, L. C. A. (2003). Crianças expostas à violência conjugal: uma revisão de área. $\ln \mathrm{M}$. C. Marquezine, M. A. S. Almeida, S. Omote, \& E. D. O. Tanaka (Orgs.), O papel da família junto ao portador de necessidades especiais (pp. 123-130). Londrina, PR: Eduel.
Brasil. (1990). Estatuto da Criança e do Adolescente. Lei n 8.069, de 13 de julho de 1990. Brasília: Ministério da Justiça.

Cherry, C. L. (2006). Animal cruelty by children exposed to domestic violence. Child Abuse \& Neglect, 30(4), 425-435.

Coker, A. L., Davis, K. E., Arias, I., Desai, S., Sanderson, M.,Brandt, H. M., \& Smith, P. H. (2002). Physical and mental health effects of intimate partner violence for men and women. American Journal of Preventive Medicine, 23(4), 260-268.

Cortez, M. B., Padovani, R. C., \& Williams, L. C. A. (2005). Terapia cognitivo-comportamental com agressores conjugais. Estudos de Psicologia, Campinas, 22(1), 13-21.

Deslandes, S. F., Gomes, R., \& Silva, C. M. F. P. (2000). Caracterização dos casos de violência doméstica contra mulher atendidos em hospitais públicos do Rio de Janeiro. Cadernos de Saúde Pública, 16(1), 129-137. 
Dong, M., Anda, R. F., Feliti, V. J., Dube, S. R., Williamson, D. F., Thompson, T. J. et al. (2004). The interrelatedness of multiple forms of childhood abuse, neglect, and household dysfucntion. Child Abuse \& Neglect, 28, 771-784.

Duran, B., Malcoe, L. H., Sanders, M., Waitzkin, H., Skipper, B., \& Yager, J. (2004). Child maltreatment prevalence and mental disorders outcomes among American Indian women in primary care. Child Abuse \& Neglect, 28(4), 131-145.

Edwards, V. J., Anda, R. F., Dube, S. R., Dong, M., Chapman, D. P., \& Feliti, V. J. (2005). The wide-ranging health outcomes of adverse childhood experiences. In K. A. Kendall-Tackett \& S. M. Giacomoni (Eds.), Child victimization: Maltreatment, bullying and dating violence. New Jersey: Civic Research Institute.

Goldberg, J. F., \& Garno, J. L. (2005). Development of posttraumatic stress disorder in adult bipolar patients with histories of severe childhood abuse. Journal of Psychiatric Research, 39, 598-601.

Gondolf, E. W. (1997). Characteristics of batterers in a multisite evaluation of batterer intervention systems-summary of the 15-month "follow-up". Indiana: Universidade da Pensilvânia.

Koss, M. P., Bailey, J. A., \& Yan, N. P. (2003). Depression and PTSD in survivors of male violence: Research and training initiatives to facilitate recovery. Psychology of Women Quarterly, 27, 130-142.

Maldonado, D. P. A., \& Williams, L. C. A. (2005). O comportamento agressivo de crianças do sexo masculino na escola e sua relação com a violência doméstica. Psicologia em Estudo, 10(3), 353-362.

Martin, G., Bergen, H. A., Richardson, A. S., \& Allison, S. (2004). Sexual abuse and suicidality: Gender differences in a large community sample of adolescents. Child Abuse \& Neglect, 28(5), 491-503.

Melo, A. P. S., \& Guimarães, M. D. C. (2005). Fatores associados ao abandono do tratamento psiquiátrico em um centro de referência em saúde mental em Belo Horizonte. Revista Brasileira de Psiquiatria, 27(2), 113-118.

Minayo, M. C. S. (2002). O significado social para a saúde da violência contra crianças e adolescentes. In M. F. Westphal (Org.), Violência e criança (pp. 95-114). São Paulo: Edusp.

Ministério da Saúde. (2007). Relatório de gestão 2003-2006: saúde mental no SUS - acesso ao tratamento e mudança do modelo de atenção. Brasília, DF: Autor.

Morrison, L. J., Allan, R., \& Grunfeld, A. (2002). Improving the emergency department detection rate of domestic violence using direct questioning. The Journal of Emergency Medicine, $19(2), 117-124$

Nolen-Hoeksema, S., \& Keita, G. P. (2003). Women and depression: Introduction. Psychology of Women Quarterly, 27, 89-90.

Olive, P. (2007). Care for emergency departament patients who have experienced domestic violence: A review of the evidence base. Journal of Clinical Nursing, 16(9), 1736-1748.

Oliveira, H. (2002). A violência doméstica contra criança e o adolescente. In M. F. Westphal (Org.), Violência e criança (pp. 137-140). São Paulo: Edusp.

Organização Mundial da Saúde. (1993). Classificação de transtornos mentais e de comportamento da CID-10. Porto Alegre: Artmed.
Ormeño, G. I. R., \& Williams, L. C. A. (2006). Intervenção precoce com crianças agressivas: suporte à família e à escola. In $\mathrm{H}$. J.Guilhardi \& N. C. Aguirre (Orgs.), Sobre comportamento e cognição (pp. 168-182). Santo André, SP: ESETec.

Padovani, R. C., \& Williams, L.C.A. (2002). Intervenção psicoterapêutica com agressor conjugal: um estudo de caso. Psicologia em Estudo, 7, 13-17.

Ramos, B. M., Carlson, B. E., \& Mcnutt, L. A. (2004). Lifetime abuse, mental health and African American woman. Journal of Family Violence, 19(3), 153-164.

Read, J. (2007). To ask, or not to ask, about abuse - New Zeland research. American Psychologist, 62(4), 325-326.

Rodrigues, J. L., Brino, R. F., \& Williams, L. C. A. (2006). Concepções de sexualidade entre adolescentes com e sem histórico de violência sexual. Paidéia, 16(34), 229-240.

Rynerson, B. C., \& Fishel, A. H. (1993). Domestic violence prevention training: Participant characteristics and treatment outcomes. Journal of Family Violence, 8(3), 253-267.

Saffioti, H. I. (1997).Violência doméstica ou a lógica do galinheiro. In M. Kupstas (Org.), Violência em debate (pp. 39-57). São Paulo: Moderna.

Santos, M. C. C. L. (2002). Raízes da violência na criança e danos psíquicos. In M. F. Westphal (Org.), Violência e criança (pp. 189-204). São Paulo: Edusp.

Santos, L. L. (2003). A visibilidade da violência de gênero em dois serviços de assistência primária à saúde. Dissertação de Mestrado, Faculdade de Medicina, Universidade de São Paulo, Ribeirão Preto, SP

Schraiber, L. B., D'Oliveira, A. F., França, I., \& Pinho, A. A. (2002). Violência contra a mulher: Estudo em uma unidade de atenção primária à saúde. Revista de Saúde Pública, 36(4), 470-477.

Sinclair, D. (1985). Understanding wife assault: A training manual for counsellors and advocates. Toronto: Publications Ontario.

Smith, C. A., Ireland, T. O., \& Thornberry,T. P. (2005). Adolescent maltreatment and its impact on young adult antisocial behavior. Child Abuse \& Neglect, 29(1), 1099-1119.

Soares, B. M. (1999). Mulheres invisíveis: violência conjugal e novas políticas de segurança. Rio de Janeiro: Civilização Brasileira.

Whitfield, C. L., Dube, S. R., Felitti, V. J., \& Anda, R. F. (2005). Adverse childhood experiences and hallucinations. Child Abuse \& Neglect, 29(7), 797-810.

Williams, L. C. A. (2001). Violência doméstica: há o que fazer? In H. J. Guilhardi, M. B. B. Madi, P. P. Queiroz, \& M. C. Scoz (Orgs.), Sobre comportamento e cognição: expondo a variabilidade (Vol. 7, pp. 112). Santo André, SP: ESETec.

Williams, L. C. A. (2002). Abuso sexual infantil. In H. J. Guilhardi, M. B. B. Madi, P. P. Queiroz, \& M. C. Scoz (Orgs.), Sobre comportamento e cognição: contribuições para a construção da teoria do comportamento (pp. 155-164). Santo André, SP: ESETec.

Williams, L. C. A. (2004). Entrevista com crianças expostas à violência conjugal. Trabalho não publicado, LAPREV, Universidade Federal de São Carlos, São Carlos, SP. 